\title{
Killing of Streptococcus pneumoniae by capsular polysaccharide-specific polymeric IgA, complement, and phagocytes
}

\author{
Edward N. Janoff, ${ }^{1}$ Claudine Fasching, ${ }^{1}$ Jan M. Orenstein, ${ }^{2}$ Jeffrey B. Rubins, ${ }^{3}$ \\ Nancy L. Opstad, ${ }^{1}$ and Agustin P. Dalmasso ${ }^{4}$ \\ ${ }^{1}$ Infectious Disease Section, Department of Medicine, Veterans Affairs Medical Center and University of Minnesota Medical School, \\ Minneapolis, Minnesota 55417, USA \\ ${ }^{2}$ Department of Pathology, George Washington School of Medicine, Washington, DC 20037, USA \\ ${ }^{3}$ Pulmonary Section, Department of Medicine, Veterans Affairs Medical Center and University of Minnesota Medical School, \\ Minneapolis, Minnesota 55417, USA \\ ${ }^{4}$ Department of Pathology and Laboratory Medicine, Veterans Affairs Medical Center and University of Minnesota Medical School, \\ Minneapolis, Minnesota 55417, USA
}

Address correspondence to: Edward N. Janoff, Veterans Affairs Medical Center, Infectious Disease Section (111F),

One Veterans Drive, Minneapolis, Minnesota 55417, USA.

Phone: (612) 725-2000 ext. 4185; Fax: (612) 727-5995; E-mail: janof001@tc.umn.edu.

Portions of this work were presented at the 34th Interscience Conference on Antimicrobial Agents and Chemotherapy in New Orleans, Louisiana, USA, on October 4-7, 1996, and at the American Association of Immunologists Annual Meeting in San Francisco, California, USA, on April 18-22, 1998.

Received for publication January 19, 1999, and accepted in revised form September 10, 1999.

The role of IgA in the control of invasive mucosal pathogens such as Streptococcus pneumoniae is poorly understood. We demonstrate that human pneumococcal capsular polysaccharide-specific IgA initiated dose-dependent killing of S. pneumoniae with complement and phagocytes. The majority of specific IgA in serum was of the polymeric form (PIgA), and the efficiency of PIgA-initiated killing exceeded that of monomeric IgA-initiated killing. In the absence of complement, specific IgA induced minimal bacterial adherence, uptake, and killing. Killing of S. pneumoniae by resting phagocytes with immune IgA required complement, predominantly via the $\mathrm{C} 2$-independent alternative pathway, which requires factor B, but not calcium. Both S. pneumoniae-bound IgA and complement were involved, as demonstrated by a $50 \%$ decrease in killing with blocking of $\mathrm{Fc} \alpha$ receptor (CD89) and CR1/CR3 (CD35/CD11b). However, IgA-mediated killing by phagocytes could be reproduced in the absence of opsonic complement by pre-activating phagocytes with the inflammatory products C5a and TNF- $\alpha$. Thus, S. pneumoniae capsule-specific IgA may show distinct roles in effecting clearance of $S$. pneumoniae in the presence or absence of inflammation. These data suggest mechanisms whereby PIgA may serve to control pneumococcal infections locally and upon the pathogen's entry into the bloodstream.

J. Clin. Invest. 104:1139-1147 (1999).

\section{Introduction}

At mucosal sites, the role of secretory IgA (sIgA) has been characterized as inhibition of microbial adherence and inflammation $(1,2)$, and neutralization of viruses, toxins, and enzymes (3-11). These local actions may serve to retard ingress of pathogens or their products into the host's internal compartment and to prevent injury of tissues and internal organs. At systemic sites, serum IgA has been reported to limit inflammation by inhibiting complement activation by IgG (12), and also to induce complement-mediated killing of Gram-negative organisms (e.g., Neisseria meningitidis and Haemophilus influenzae type B) (13). However, whether pathogen-specific IgA also plays a role in limiting or enhancing killing of invasive Grampositive bacteria by phagocytes has not been determined.

We recently reported that up to a third of serum antibodies that are reactive with the polysaccharide capsule of an invasive mucosal Gram-positive organism, Streptococcus pneumoniae, are of the IgA class $(14,15)$. The majority of this pathogen-specific IgA in serum is in the polymeric form ( $\geq 2$ IgA molecules bound by a J chain) and remains polymeric long after immunization or infection (15), even though about $90 \%$ of the total IgA in serum is in the monomeric form (5). S. pneumoniae may colonize the nasopharyngeal mucosa without sequelae but also commonly causes serious invasive clinical syndromes, including pneumonia, bacteremia, and meningitis in children and adults (16-19). Natural infection and immunization elicit capsule-specific polymeric IgA (pIgA) responses in blood and at mucosal sites $(14,15,20-22)$. Thus, pIgA may play a functional role at both mucosal and systemic sites.

Therefore, we determined levels, specificity, structure, and the functional activity of serum IgA that reacts with pneumococcal capsular polysaccharide in response to antigenic challenge. We found that immune IgA bound specifically to the capsule. However, IgA-associated binding, uptake, and killing of S. pneumoniae by phagocytes required either opsonization by complement in human serum, predominantly by the alternative pathway, or prior activation of phagocytes by TNF- $\alpha$ and C5a. That the polymeric form 
of antigen-specific IgA (also found at mucosal sites as sIgA) may bind to the capsule of $S$. pneumoniae, but mediates uptake and killing of this invasive Gram-positive organism only in the presence of inflammatory products, suggests a dichotomous role of pathogen-specific IgA in quiescent and inflamed environments.

\section{Methods}

Subjects. Informed consent was obtained from all study participants according to protocols approved by the Human Subjects Subcommittees at the Minneapolis Veterans Affairs Medical Center and the University of Minnesota.

Sera were obtained from 5 healthy human volunteers ( 3 male and 2 female, aged 24-36 years) before and 4 weeks
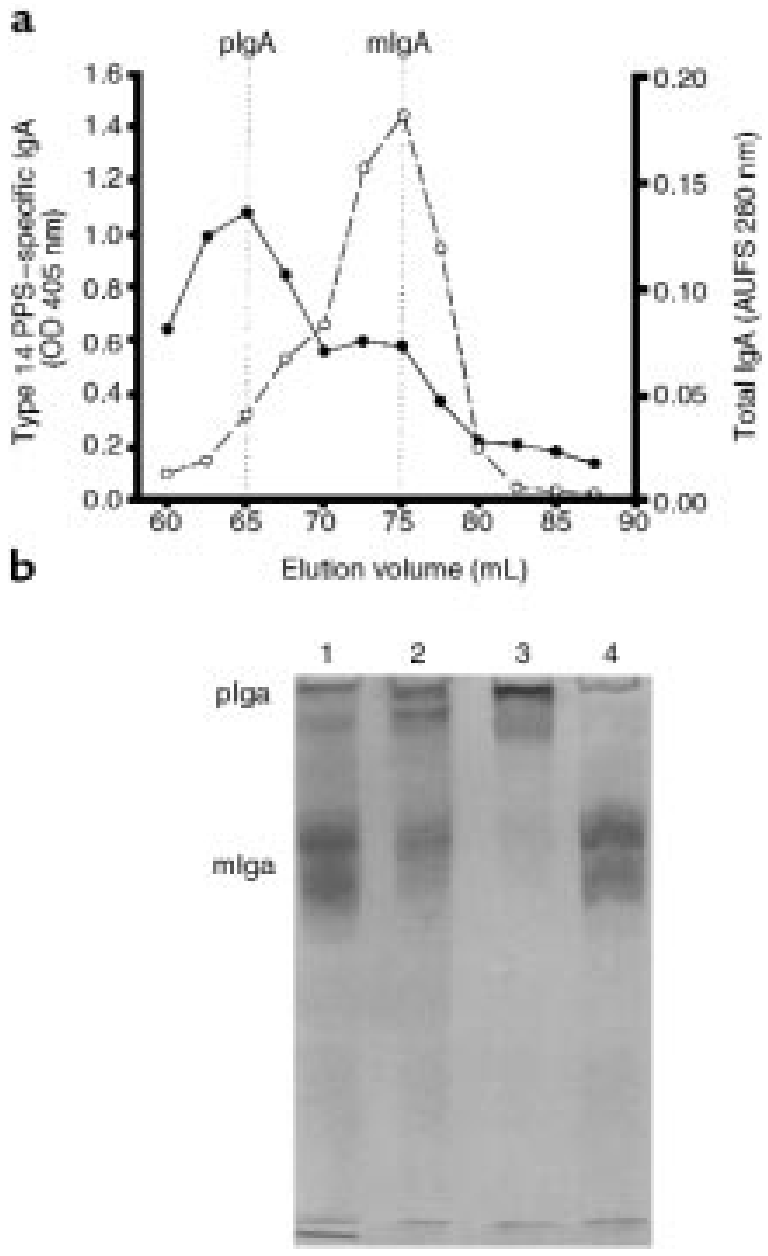

\section{Figure 1}

(a) Distribution of molecular forms of $\operatorname{lgA}(\mathrm{mlgA}$ and $\mathrm{plgA}$ ) in immune serum for type 14 PPS-specific IgA (black circles; solid lines) and total serum IgA (open circles; dotted line) by gel filtration (mean of 2 experiments). Optical density (OD) represents absorbance at $405 \mathrm{~nm}$ by ELISA for type 14-specific IgA and absorbance at $280 \mathrm{~nm}$ for total serum IgA (0.2 AUFS absorbance units, full scale); the latter represents approximately 1,000 -fold more protein than the former $(2 \mu \mathrm{g} / \mathrm{mL}$ type 14 -specific IgA and $2 \mathrm{mg} / \mathrm{mL}$ total IgA). Type 14 -specific IgA comprised $1.2 \%$ of plgA and $0.15 \%$ of mlgA. Vertical lines represent peaks of plgA and mlgA standards. (b) Nondenaturing PAGE of purified IgA before and after size fractionation by gel filtration. Lane 1: purified IgA from serum sample before separation; lane 2: IgA standard containing both plgA and $\mathrm{mlgA}$; lane 3: purified plga fraction pool; lane 4: purified mlgA pool. after intramuscular immunization with 23-valent capsular pneumococcal polysaccharide (PPS) vaccine (PNUIMMUNE (23); a generous gift from Lederle Labs Division of American Cyanamid Company, Pearl River, New York, USA). Sera were frozen at $-20^{\circ} \mathrm{C}$ until tested.

Purification of serum IgG and IgA. IgG was purified from whole sera using a Protein G Hi-Trap column (Amersham Pharmacia Biotech, Piscataway, New Jersey, USA) as described previously (15), followed by IgM depletion; subsequently, IgA was purified from the non-IgG, non-IgM fraction. IgM and IgA affinity columns were prepared with goat anti-human IgM or IgA (Southern Biotechnology Associates, Birmingham, Alabama, USA) coupled to CNBractivated Sepharose 4B (Amersham Pharmacia Biotech) as described (15). The IgA fractions $(n=7)$ contained 97.5-99.9\% IgA, 0.1-2\% IgM, less than 0.1-0.2\% IgG, and no detectable type 14 capsule-specific IgG or IgM.

Total and PPS-specific Ig. Levels of total IgA, IgM, and IgG were measured by ELISA with unlabeled and labeled affinity-purified goat anti-human IgA, IgM, or IgG (Jackson ImmunoResearch Laboratories Inc., West Grove, Pennsylvania, USA) as the capture and detector antibodies, respectively (15). Capsule-specific antibodies against type 14 PPS were measured by ELISA as described $(14,15,23)$ after samples and standards were preadsorbed overnight at $4{ }^{\circ} \mathrm{C}$ with purified pneumococcal cell wall polysaccharide (Statens Serum Institut, Copenhagen, Denmark) at a final concentration of 50 $\mu \mathrm{g} / \mathrm{mL}$. The specificity of the ELISA for IgA against type 14 capsule was established with a competitive inhibition assay $(24,25)$. Increasing concentrations (0.1-100 $\mu \mathrm{g} / \mathrm{mL}$ ) of both homologous (type 14 pneumococcal capsule) and heterologous soluble antigens (type 4, 8, and 19A pneumococcal capsule; H. influenzae type B capsule [poly-3-D-ribose(1-1)ribitol-5 phosphate]; and tetanus toxoid) were incubated overnight at $4^{\circ} \mathrm{C}$ with a fixed concentration of specific IgA (optical density of 1.0 unit at $410 \mathrm{~nm}$ ). The ability of these soluble antigens to inhibit binding of IgA to solid-phase type 14 capsule by ELISA after a 2-hour incubation at $25^{\circ} \mathrm{C}$ was compared. IgA fractionation by molecular form. Purified IgA was fractionated as described previously $(8,15)$ by molecular sieve chromatography using a Sephacryl S-300 HR column (Pharmacia Biotech Inc., Piscataway, New Jersey, USA) that was calibrated with pIgA and monomeric IgA (mIgA) standards (generous gifts of J. Mestecky, University of Alabama, Birmingham, Alabama, USA). The polymeric structure of the capsule-specific IgA did not change with acid treatment (15), which dissociates immune complexes but not pIgA $(26,27)$, but did dissociate to IgA monomers under partial reducing conditions $(8,15,27,28)$. The purity of $\mathrm{pIgA}$ and $\mathrm{mIgA}$ fractions was confirmed by resolution on a $5 \%$ continuous nondenaturing polyacrylamide gel stained with Coomassie blue. The absence of secretory component in association with purified IgA fractions was determined by ELISA using anti-human IgA or purified pneumococcal type 14 capsular polysaccharide, as described above, and horseradish peroxidase-labeled antisecretory component (DAKO Corp., Carpinteria, California, USA).

Antibody-dependent killing of S. pneumoniae by complement and phagocytes. Purified IgA or control IgG were incubat- 
ed with shaking for 30 minutes at $25^{\circ} \mathrm{C}$ with $1,000 \mathrm{CFU}$ of log-phase type 14 S. pneumoniae (American Type Culture Collection 6314, Rockville, Maryland, USA) (29-31). Phagocytes $\left(4 \times 10^{5}\right)$ and a specified human or rabbit serum complement source $(10 \%)$ were added, incubated at $37^{\circ} \mathrm{C}$ for 1 hour with shaking, and lysed in distilled water (1:10); serial dilutions were then plated. Phagocytes were either freshly isolated peripheral blood neutrophils (PMN) or cultured HL-60 cells (Certified Cell Line 240; American Type Culture Collection) treated with $120 \mathrm{mM}$ dimethyl formamide to induce differentiation $(31,32)$. We verified the absence of $\operatorname{IgG}$ and $\operatorname{IgA}$ bound to purified human neutrophils by FACS $^{\circledR}$ with affinity-purified FITC-labeled goat $\mathrm{F}\left(\mathrm{ab}^{\prime}\right)_{2}$ anti-human $\mathrm{Fc} \gamma$ and $\mathrm{Fc} \alpha$ (Southern Biotechnology Associates), respectively. Cells were also washed 3 times to remove soluble Igs from the donor. To determine the antibody specificity, killing assays were performed with and without preincubation of purified IgG and IgA with $20 \mu \mathrm{g} / \mathrm{mL}$ cell wall polysaccharide and type 14 PPS; control wells contained 10\% heat-inactivated FCS (GIBCO BRL, Grand Island, New York, USA). Killing activity of antibodies with complement and phagocytes (or with activated phagocytes and no complement) was calculated using the following formula: percent kill $=[($ bacteria present with no Ig - bacteria present with $\operatorname{IgG}$ or IgA)/bacteria present with no Ig] $\times 100$. To determine the receptors involved in killing, selected experiments were performed with preincubation of phagocytes with isotype controls (Southern Biotechnology Associates and PharMingen, San Diego, California, USA) and unlabeled antibodies with blocking activity against complement receptor CR1 (CD35; DAKO Corp.), CR3 (CD11b; BioSource International, Camaril- lo, California, USA), Fc $\alpha$ receptor (CD89; Medarex Inc., Annandale, New Jersey, USA), Fc $\gamma$ receptor I (CD64; PharMingen), Fc $\gamma$ receptor II, and Fc $\gamma$ receptor III (CD32 and CD16; Medarex Inc.).

Sources and treatment of complement. Complement sources were baby rabbit serum (Cedarlane Laboratories Ltd., Hornby, Ontario, Canada) that was shown to be free of antipneumococcal activity, or human serum from a hypogammaglobulinemia patient (serum Ig in $\mathrm{mg} / \mathrm{mL}$ : IgG, <2; IgM, $<0.4$; and IgA, <0.07), or human complement depleted of C2 or factor B (QUIDEL Corp., San Diego, California, USA). Human complement was adsorbed twice with $10^{9} \mathrm{CFU} S$. pneumoniae per mL serum with $25 \mathrm{mM}$ EDTA for $30 \mathrm{~min}$ utes on ice, and then dialyzed and filter-sterilized before use. Adsorbed complement sources retained complement activity and lacked detectable (by ELISA) antibodies to capsular type 14 . Selected assays were performed in the presence of $10 \mathrm{mM}$ EDTA (to block both classical and alternative pathways), $10 \mathrm{mM} \mathrm{MgEGTA} \mathrm{(to} \mathrm{selectively} \mathrm{inhibit} \mathrm{the} \mathrm{calcium-}$ dependent classical pathway) (33), or factor B-depleted serum reconstituted with factor B (QUIDEL Corp.).

Receptor expression. The expression of receptors for C3bi (CR3/CD11b) and for IgA (Fc $\alpha$ receptor CD89) on neutrophils and HL-60 cells was examined by flow cytometry with mAbs specific for CR3 (Becton Dickinson Immunocytometry Systems, San Jose, California, USA), Fc $\alpha$ receptor (PharMingen), and isotype and fluorochrome controls.

Analysis of uptake of S. pneumoniae by phagocytes. Specified concentrations of antibody (purified IgA or IgG) and $S$. pneumoniae $\left(2 \times 10^{5} \mathrm{CFU}\right)$ were combined in a total volume of $50 \mu \mathrm{L}$ and shaken in Immulon I wells (Dynex Technologies, Chantilly, Virginia, USA) for 30 minutes at $25^{\circ} \mathrm{C}$. Baby rabbit complement and purified neutrophils $\left(2 \times 10^{5}\right.$
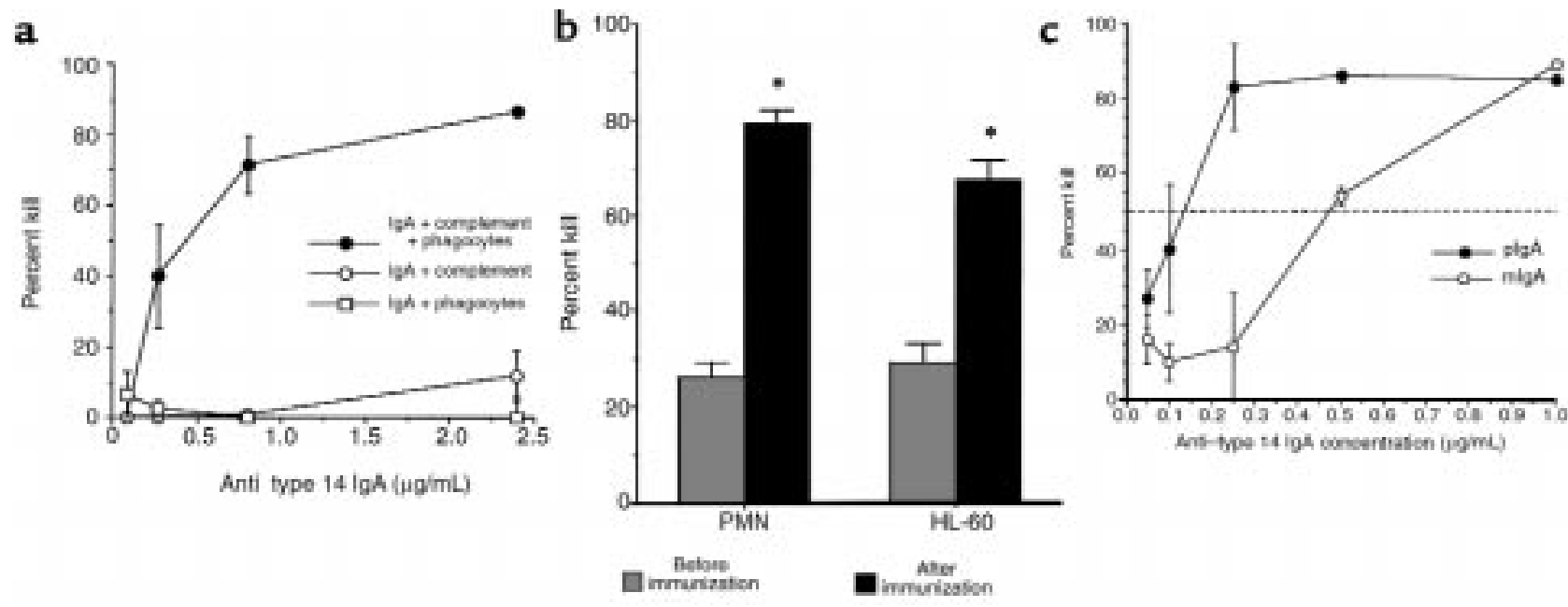

Figure 2

(a) IgA-mediated killing of type 14 S. pneumoniae by differentiated HL-60 cells and baby rabbit complement. Phagocyte/bacteria ratio was 400:1. Tests were run in duplicate; results are shown as the mean \pm SEM of 3 experiments. (b) Killing of type 14 S. pneumoniae by human polymorphonuclear leukocytes (PMN) and differentiated HL-60 cells in the presence of $10 \%$ baby rabbit complement and $1.2 \mathrm{mg} / \mathrm{mL}$ of total $\lg A(98 \% \lg A,<1 \% \lg G,<2 \% \lg M)$. Given in Results are means \pm SEM of experiments (PMN, $n=4 ; \mathrm{HL}-60$ cells, $n=6$ ) with IgA purified from serum of volunteers before and 1 month after immunization with 23-valent pneumococcal vaccine. Levels of type 14-specific IgA were $678 \mathrm{ng} / \mathrm{mL}$ before immunization and 2,445 ng/mL after immunization in these pools of purified IgA. ${ }^{*} P<0.0001$ for percent kill before immunization vs. 1 month after immunization. (c) The efficacy of molecular forms of IgA on phagocytic killing of type 14 S. pneumoniae. Serial dilutions of purified mlgA and plgA were tested in the presence of $10 \%$ baby rabbit complement for their ability to mediate complement-dependent killing of the organism. Percent kill is given as a function of the concentration of anti-type $14 \lg A$ in purified $\mathrm{mlgA}$ and plgA. Tests were run in duplicate; results are shown as the mean \pm SEM of 2 experiments. 


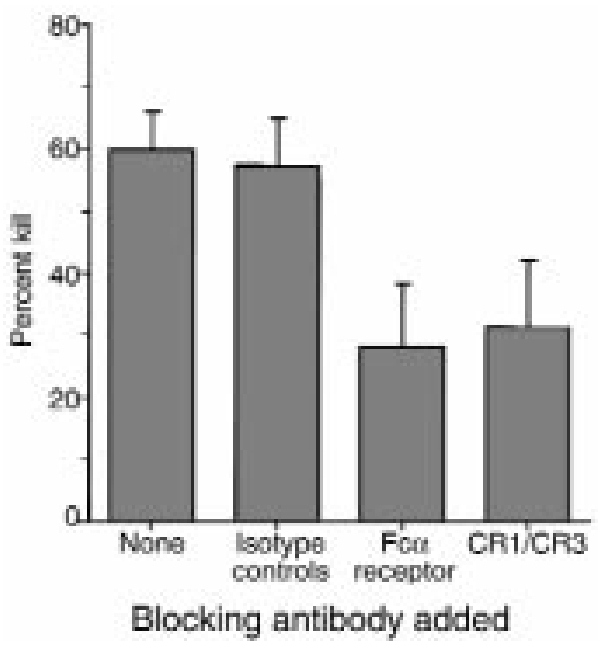

\section{Figure 3}

Effect of receptor-blocking mAbs and isotype control antibodies on killing of type $14 \mathrm{~S}$. pneumoniae with $2 \mu \mathrm{g} / \mathrm{mL}$ specific $\lg A$ and $10 \%$ baby rabbit complement. Differentiated HL-60 cells were preincubated for 30 minutes at $25^{\circ} \mathrm{C}$ with blocking antibodies $(40 \mu \mathrm{g} / \mathrm{mL})$ before addition of IgA-opsonized organisms and complement. Results are shown as the mean \pm SEM of 2 experiments. $P=0.08$ for percent kill with anti-Fc $\alpha$ receptor antibody compared with killing without blocking antibody.

per well) were added, and the final mixture $(100 \mu \mathrm{L})$ was incubated at $37^{\circ} \mathrm{C}$ for 1 hour with shaking. Well contents were either removed for cytospin (Cytospin 2, Shandon Inc., Pittsburgh, Pennsylvania, USA) or slow spun at $150 \mathrm{~g}$ to pellet neutrophils. Cytospins were stained with WrightGiemsa for light microscopy. Cell pellets were fixed in 2.5\% glutaraldehyde and processed for transmission electron microscopy as described (34). In 3 experiments, whole complement was excluded and neutrophils were preactivated with $10^{-8} \mathrm{M}$ recombinant human TNF- $\alpha$ (R\&D Systems Inc., Minneapolis, Minnesota, USA), purified $10^{-9} \mathrm{M}$ C5a (CalBiochem-Novabiochem Corp., San Diego, California, USA), or both agents together.

Statistics. Mean levels of capsule-specific IgA and percent kill after immunization were compared with preimmunization levels by paired 2-tailed $t$ tests. Data were analyzed using the StatView 4.0 statistical program (Abacus Concepts Inc., Berkeley, California, USA).

\section{Results}

Measurement of type 14 capsule-specific IgA in serum. Levels of IgA that were reactive with PPS 14 measured by ELISA increased 13-fold in sera from 5 subjects 1 month after immunization $(0.15 \pm 0.02 \mu \mathrm{g} / \mathrm{mL}$ before immunization vs. $2.1 \pm 0.3 \mu \mathrm{g} / \mathrm{mL}$ after immunization). The IgA detected was known to be specific for the polysaccharide capsule because its binding was inhibited by preadsorption with soluble homologous antigen (PPS 14) but not by other heterologous antigens (not shown). The majority of PPS 14-specific IgA was in the polymeric rather than the monomeric form, even though $80-90 \%$ of total IgA in serum is mIgA (Figure 1, a and b). Capsule-specific IgA comprised approximately $1.2 \%$ of total pIgA but only $0.15 \%$ of total mIgA (an 8 -fold difference). Both total and capsule-specific pIgA had little to no secretory component detectable by ELISA (not shown). As reported previously, $80-90 \%$ of capsule-specific IgA is of the IgA1 subclass 1 month after immunization (14).

IgA-associated killing of S. pneumoniae. In the presence of complement and phagocytes, immune IgA mediated killing of the organism (Figure 2a). Killing increased with the concentration of immune IgA, required the presence of complement, and increased significantly after immunization (Figure 2b). Activity was highly correlated with PPS 14-specific IgA levels, as measured by ELISA (results not shown). IgA-associated killing of $S$. pneumoniae was caused by reactivity with the capsule killing was inhibited $51 \%$ by preadsorption with 20 $\mu \mathrm{g} / \mathrm{mL}$ capsular polysaccharides (PPS 14) but not with $20 \mu \mathrm{g} / \mathrm{mL}$ cell wall polysaccharides. Purified pIgA showed greater killing activity in the presence of complement and PMN than did MIgA (Figure 2c), although activity was similar between the 2 forms at high concentrations. The efficiency of capsule-specific PIgA was greater than that of specific mIgA, with 50\% kill achieved with $0.13 \mu \mathrm{g} / \mathrm{mL}$ pIgA, and $0.46 \mu \mathrm{g} / \mathrm{mL}$ mIgA.

Killing of S. pneumoniae was similar with both PMN and differentiated HL-60 cells (Figure 2a), each of which expressed receptors for IgA (Fc $\alpha$ receptor CD89; 91\% and $93 \%$, respectively) and CR3 (CD11b/18; 78\% and 100\%, respectively). The density of receptor expression measured by mean fluorescence intensity was similar for the 2 cell types for CD89, whereas CR3 expression was higher on PMN cells than on differentiated HL-60 cells (mean fluorescence intensity $=202$ and 99 , respectively). Both Fc $\alpha$ receptor and CR1/CR3 contributed to IgAmediated killing by phagocytes, as demonstrated by the fact that blocking of these receptors by specific mAbs reduced killing by about half (Figure 3). Addition of both sets of blocking antibodies simultaneously did not further inhibit killing (not shown); addition of antibodies to Fc $\gamma$ receptors I, II, and III had no effect on IgA-initiated killing (not shown). Moreover, undifferentiated HL-60 cells (which expressed neither Fc $\alpha$ receptor nor CR1/CR3) effected no killing of the organism in the presence of complement and immune IgA or IgG.

Role of complement. Despite high levels of capsule-specific IgA detected by ELISA, immune IgA in the absence of complement mediated little or no adherence of organisms to PMNs as detected by light microscopy (Table 1). In con-

\section{Table 1}

Bacterial adherence to PMNs with and without complement by light microscopy of Wright-Giemsa-stained cytospin preparations

\begin{tabular}{ccc}
\hline Complement & Antibody & $\begin{array}{c}\text { Percent of PMNs with } \\
\text { associated bacteria }\end{array}$ \\
- & - & $4.3^{\mathrm{A}} \pm 2.0$ \\
+ & - & $9.7^{\mathrm{A}} \pm 0.7$ \\
- & $\lg A$ & $9.0^{\mathrm{A}} \pm 7.1$ \\
+ & $\lg A$ & $58.3^{\mathrm{B}} \pm 14.8$ \\
- & $\lg \mathrm{G}$ & $63.5^{\mathrm{B}} \pm 12.5$ \\
+ & $\lg \mathrm{G}$ & $97.5^{\mathrm{B}} \pm 1.5$ \\
\hline
\end{tabular}

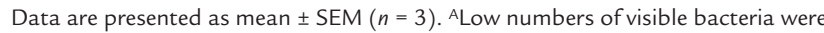
predominantly adjacent to external cell membrane, not internalized or in vacuoles. BThe majority of visible bacteria were internalized, as confirmed by transmission electron microscopy. Type 14-specific antibody concentration was $1.9 \mu \mathrm{g} / \mathrm{mL}$ in purified $\lg A$ and $0.7 \mu \mathrm{g} / \mathrm{mL}$ in purified $\lg \mathrm{G}$. 


\section{Figure 4}

Transmission electron microscopy of preparations treated with baby rabbit complement and IgA (a-c) or IgG (d). (a) The bacteria in the 3 phagosomes of a neutrophil are surrounded by electron-dense flocculent material. An empty shell is apparently all that remains of 1 bacterium (arrow). $\times 12,000$. (b) Higher magnification of bacteria with coronas, in vacuoles surrounded by primary lysosomes of varying densities (arrowheads). $\times 25,000$. (c) Still higher magnification shows changes in the integrity of a bacterium and membranous material that probably represents bacterial debris (arrow). $\times 50,000$. (d) Three vacuoles are surrounded by electron-dense lysosomes and contain lysosomal constituents, some encasing bacteria, and bacterial debris (arrows). $\times 16,000$.

trast to results with $\operatorname{IgA}$, control experiments showed that purified immune IgG was sufficient to induce both cell association and uptake of the organism into phagosomes. In the presence of complement, IgA mediated adherence, uptake, and dose-dependent killing of the organisms by PMNs (Table 1; Figure 2a). The presence of complement with both IgA and IgG increased binding and uptake of S. pneumoniae into phagosomes with lysosomal activity, bacterial destruction, and killing (Table 1; Figure 4).

As shown by transmission electron microscopy, in the absence of antibody and complement, only rare cells had single bacteria associated with their surfaces. In the presence of complement or IgA alone, only a few cells showed 1 or 2 large vacuoles containing individual normal-appearing bacteria, and primary lysosomes were not concentrated in the adjacent cytoplasm (not shown). Without complement, heat-inactivated immune serum or IgG led to substantially more bacterial uptake than with IgA, but virtually without evidence of fusion of primary lysosomes with phagosomes or alteration in the appearance of the bacteria. In fact, bacteria still appeared to be capable of replication while within cytoplasmic vacuoles. In the presence of complement, about twice as many PMNs contained organisms when bacteria were exposed to immune serum or IgG as when bacteria were treated with purified IgA (Table 1), but their morphological changes were qualitatively identical (Figure 4). With complement and either IgA or IgG, bacteria were found both within loose- and tight-fitting vacuoles containing electron-dense flocculent material that appeared to derive from fusion of the phagosome with primary lysosomes. The variability of electron density of the internalized bacteria suggested progressive digestion; the laminated membranous material seen in some vacuoles probably represented bacterial debris.

Complement pathway in IgA-associated killing of S. pneumoniae. Killing of IgA-opsonized pneumococci by PMNs was predominantly related to the presence of an intact alternative complement pathway. In the presence of divalent cation chelators with either baby rabbit complement or human serum from a hypogammaglobulinemic adult (both preadsorbed with whole bacteria to remove any specific antibody), IgA-associated killing of S. pneumoniae showed significant inhibition in the presence of EDTA, but
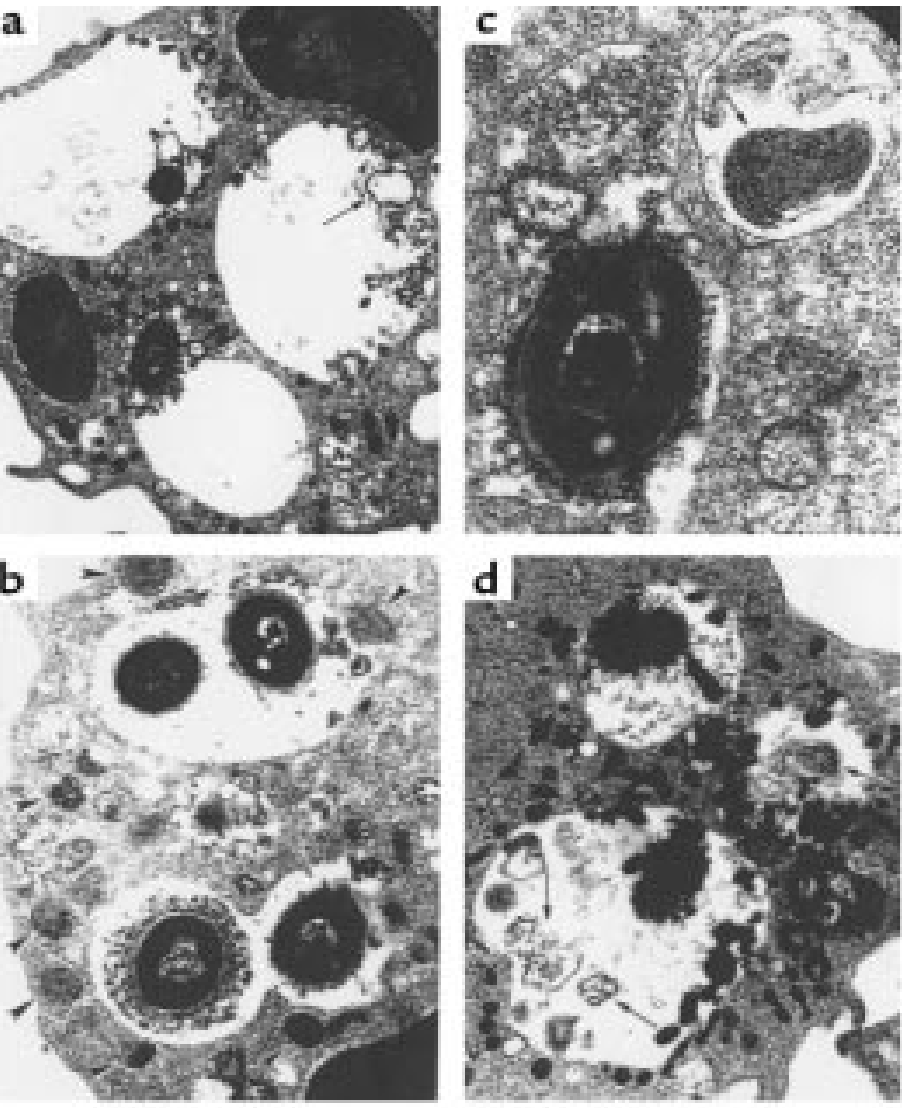

not with MgEGTA (Figure 5). Whereas EDTA chelates $\mathrm{Ca}^{2+}$ and $\mathrm{Mg}^{2+}$ and blocks activation of both the classical and alternative pathways, MgEGTA selectively chelates $\mathrm{Ca}^{2+}$ and blocks only the classical pathway. In contrast to the findings with IgA-mediated killing, IgG-associated killing was inhibited by both EDTA and MgEGTA (data not shown). Consistent with these data, depletion of a specific component of the alternative pathway, factor $\mathrm{B}$, from a human serum complement source resulted in significantly reduced killing of the organism with IgA and phagocytes compared with killing using whole complement (Figure 6). In contrast, selective impairment of the classical pathway in C2-depleted complement showed no significant decrease in killing with IgA. Reconstitution of factor B-deficient complement with purified factor B resulted in restoration of the ability to kill the organism. The possibility that residual IgG or IgM in the purified IgA preparation may partially account for the obtained results was excluded. First, the IgA preparation contained no type 14 capsule-specific IgG or IgM that was detectable by ELISA (not shown). Second, IgM purified from immune serum had no significant killing activity when tested at levels similar to those contaminating the purified IgA (not shown). Third, the killing efficiency of IgA preparations containing less than $0.1 \% \operatorname{IgM}$ was similar to those containing up to $2.5 \% \operatorname{IgM}$ (Figure 5). Fourth, no IgG was detected in the IgA fractions (limit of detection, <0.1\%). Finally, complement activation by IgM typically involves the classical complement pathway, whereas as noted above, the results with IgA involved the alternative pathway.

Killing of S. pneumoniae with IgA and activated PMNs. To determine whether IgA was capable of initiating killing of S. pneumoniae in the absence of opsonic complement 


\section{Figure 5}

Effect of cation chelators on phagocytic killing of type 14 S. pneumoniae by IgA in the presence of baby rabbit complement (a) and human complement (b). EDTA, which blocks both alternative and classical pathways, and MgEGTA, which blocks only the classical pathway, were added to killing reactions to determine the complement activation pathway involved in IgA-mediated phagocytic killing of pneumococci by differentiated HL-60 cells. Chelators (EDTA and MgEGTA) were added at $10 \mathrm{mM}$. All tests were run in triplicate; results are shown as the mean \pm SEM of 6 experiments. ${ }^{*} P<0.05$ vs. killing without EDTA. Results were the same whether individual experiments used IgA that was $98 \%$ pure $(<2 \% \lg M)$ or $99.9 \%$ pure $(<0.1 \% \lg M)$.
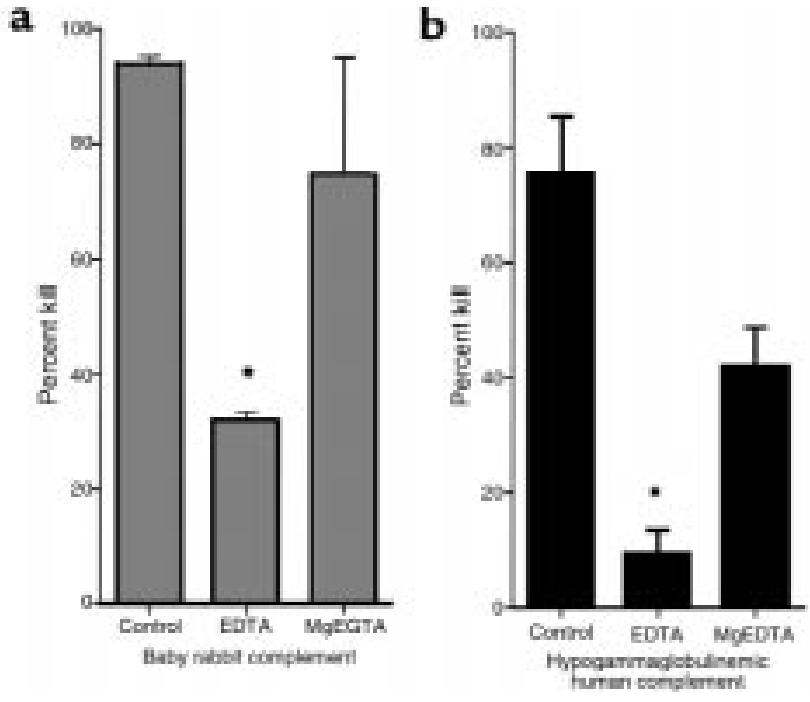

(C3bi), PMNs were activated by preincubation with products of inflammation C5a and TNF- $\alpha$. PMNs activated with C5a and TNF- $\alpha$ killed $S$. pneumoniae that was opsonized with IgA at levels comparable to those with opsonic complement and IgA (Figure 7). Again, IgA alone mediated little killing by nonactivated phagocytes. Exposure of PMNs to C5a, TNF- $\alpha$, or both did not result in spontaneous killing of the organisms in the absence of IgA (data not shown). The additive effects of C5a and TNF- $\alpha$ on IgA-associated killing may be related to the concentrations used. By light microscopy of cytospin preparations, the frequency of association of S. pneumoniae with PMNs paralleled the percent kill with each condition (data not shown).

\section{Discussion}

We demonstrate that the ability of capsule-specific IgA to mediate killing of $S$. pneumoniae by phagocytes is associated primarily with the polymeric molecular form of the antibody and participation of the complement system. Such activity promotes binding and uptake of the organism, as shown by light and electron microscopy, as well as killing of the organism. IgA-mediated killing of pneumococci by phagocytes (differentiated HL-60 cells and resting PMNs) was almost totally complementdependent and was decreased by blocking receptors for either IgA (Fc $\alpha$ receptor) or for C3 fragments (CR1 and CR3). However, we also report that immune IgA can mediate uptake and killing of the organism by activated PMNs in the absence of opsonic complement. Thus, IgA may serve an active functional role in the control of pneumococcal disease in the setting of an inflammatory immune response to infection.

Previous findings have shown a significant IgA response to pneumococcal capsular polysaccharides after immunization $(14,20,21,35)$ and natural infection $(15,22)$. Indeed, IgA comprises an appreciable proportion of capsule-specific IgA in immune serum $(14,15)$, but the functional activities of these antibodies had not been characterized. The antigen specificity of the IgAassociated killing of S. pneumoniae is a novel feature of our results. Purified human IgA can facilitate killing of another Gram-positive organism, Group B streptococcus, by phagocytes (36). However, IgA binding to that organism was shown to be mediated by bacterial surface protein B, which attaches to the IgA Fc fragment, rather than the antigen-specific Fab fragment of $\operatorname{IgA}(37,38)$. In contrast, $\mathrm{Fc}$ binding to the organism is an unlikely mechanism in the case of S. pneumoniae because IgA obtained before immunization showed little reactivity with the capsule and supported little phagocytic killing, whereas after immunization, both binding by ELISA and killing activity increased and were capsule-specific in competitive inhibition experiments.

Similarly, a surface protein of S. pneumoniae, SpsA, can bind to the secretory component of sIgA, but not to PIgA that lacks the secretory component $(39,40)$. The capsulespecific pIgA we describe in this report lacks secretory component, and therefore should not bind SpsA. In related systems, human sIgA was shown to inhibit murine mucosal infection with Group A streptococcus, but there was no bactericidal activity in vitro, despite the presence of phagocytic cells and complement (41). In that particular study, the titers of specific sIgA were low and may have been insufficient to support killing of the organism. Thus, our studies are among the first to confirm killing of a Grampositive organism initiated by antigen-specific IgA.

The functions of the 3 distinct molecular configurations of IgA (monomeric, polymeric, and secretory) may vary. mIga comprises the majority of total IgA in serum. pIga, a union of 2 mIgA molecules joined by a J chain, comprises a minority of total IgA but the majority of pneumococcal capsule-specific IgA in serum. Indeed, capsule-specific IgA comprised a greater proportion of $\mathrm{PIgA}$ than of mIgA. A human IgA mAb, produced in both monomeric and polymeric forms, has been described that reacts with type 8 S. pneumoniae and mediates a low level of phagocyte binding in the absence of complement (42). The same antibody induced significant protection of mice when administered systemically, although the mechanism of protection was not documented. In our studies, polymeric rather than monomeric S. pneumoniae-specific IgA 


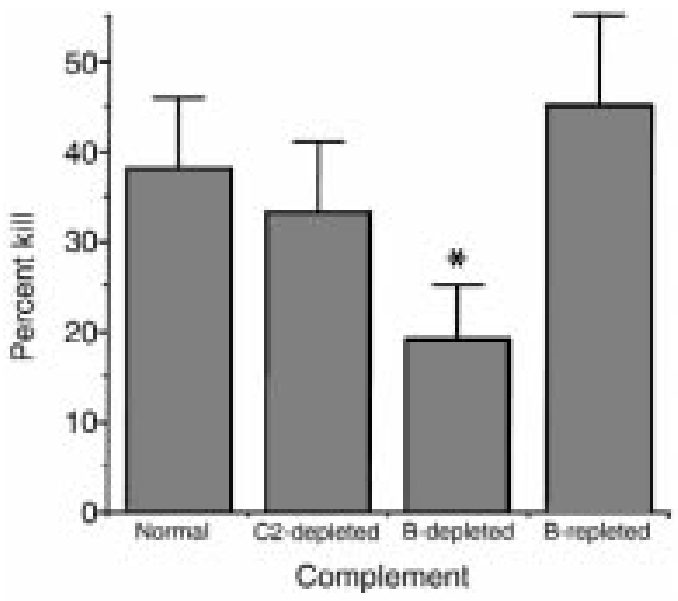

Figure 6

Effect of depletion and repletion of selected human complement components in human serum on IgA-mediated killing of type 14 S. pneumoniae by $\lg A$. Organisms were incubated with $1 \mathrm{mg} / \mathrm{mL}$ of immune IgA, differentiated HL-60 cells, and a complement source consisting of $10 \%$ serum from a hypogammaglobulinemic patient, serum depleted of the classical pathway component $C 2$, serum depleted of the alternative pathway component factor $B$, or serum depleted for factor $B$ and then repleted with a physiologic concentration of factor $B(200 \mu \mathrm{g} / \mathrm{mL})$. All sera were preadsorbed with type 14 pneumococci before the killing assay to remove specific antibody in the complement source. Results are shown as mean \pm SEM of 7 experiments. ${ }^{*} P<0.05$ vs. normal complement and factor B-repleted complement.

appears to most efficiently initiate complement-mediated killing of the organism.

This complement activation is in agreement with previous observations on complement-mediated effects of IgA in other model systems (43-45). However, we also found that mIgA can significantly mediate complementdependent killing of S. pneumoniae, albeit at a much higher IgA concentration than is required by the polymeric form. This observation suggests that the surface distribution of the capsular polysaccharide on $S$. pneumoniae may favor binding of contiguous IgA molecules that create conditions for IgA-mediated complement activation. The biochemical mechanism of IgA-initiated complement activation is not yet well defined. The third form of IgA, sIgA, accounts for almost all IgA at mucosal sites and is formed by the union of PIgA with the epithelial cell-derived secretory component (the pIgA receptor). Both PIgA and sIgA may have similar functions that extend beyond those of serum mIgA $(2,46,47)$. Our results are consistent with other functional studies of IgA that showed that, compared with mIgA, enhanced antiviral activity was demonstrated by both PIgA and SIgA (46).

We also found that IgA-mediated killing of S. pneumoniae by previously resting phagocytes depends primarily on the alternative complement pathway. Killing is inhibited by EDTA, which blocks activation of both the classical and alternative pathways; however, killing proceeds in the presence of MgEGTA, which suppresses the classical pathway but does not interfere with alternative pathway activation (33). Similarly, depletion of factor B of the alternative pathway inhibits killing by phagocytes with IgA, and repletion of factor B restores killing; depletion of the classical pathway component $\mathrm{C} 2$ has a limited effect. The variability in percent kill that we observed in experiments performed in the absence of functional complement may reflect low levels of PMN activation induced by the cell isolation procedures. Our finding that IgA activates complement via the alternative pathway is in agreement with the observations that IgA lacks the core $\mathrm{C} 1 \mathrm{q}$ binding motif of $\operatorname{IgG}$ and $\operatorname{IgM}$ (which is associated with initiation of the classical pathway [48]), and that PIgA lacks the ability to activate the classical complement pathway (49). Although high concentrations of complement alone, such as those found in serum, may promote killing of $S$. pneumoniae in the absence of antibody $(50,51)$, levels of complement at mucosal sites are much more limited (52). At mucosal sites, complement-dependent killing may require the participation of specific antibodies such as PIgA (in its secretory form). Human sIgA with specificity for type B red cells has been shown to induce complement-mediated opsonization and lysis of these cells (53). In that study and others, both PIgA and sIgA have been proposed to activate the complement cascade by the alternative pathway under certain conditions $(2,43,54-57)$.

Complement components may be produced locally by activated monocytes, mucosal macrophages (58-62), mucosal epithelial cells $(63,64)$, or recruited granulocytes (65) to support antibody-mediated killing of $S$. pneumoniae. Activation of these cells requires local inflammation, which may also contribute to exudation of serum proteins such as complement. Alternatively, local inflammation and associated phagocyte activation may abrogate the need for high levels of complement to

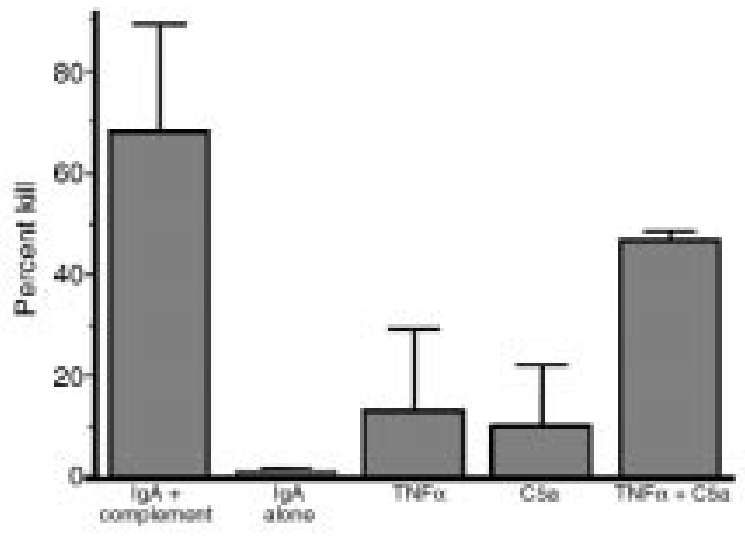

\section{Figure 7}

Effect of PMN pretreatment on IgA-mediated killing of type 14 S. pneumoniae. Human $\mathrm{PMN}$ s were preincubated with media alone (first 2 columns) or TNF- $\alpha\left(10^{-8} \mathrm{M}\right), \mathrm{C} 5 \mathrm{a}\left(10^{-9} \mathrm{M}\right)$, or both for 30 minutes before addition of log-phase organisms and purified immune $\operatorname{lgA}(1 \mathrm{mg} / \mathrm{mL}$ total $\lg A$ ). Baby rabbit complement was added to untreated PMNs with $\lg \mathrm{A}$ as a positive control (first column); IgA with untreated PMNs and no complement served as a negative control (second column). PMNs preincubated with TNF- $\alpha$ and C5a showed no uptake or killing of the organism in the absence of immune IgA (not shown). Results are shown as mean \pm SD for 3 experiments. 
effect IgA-mediated killing of mucosal pathogens.

That binding and uptake of $S$. pneumoniae is low in the absence of complement may reflect a selective 2-tiered role of IgA in immune defense. In the absence of inflammation, IgA may limit binding of pathogenic organisms, including $S$. pneumoniae, to environmentally exposed host mucosal tissues, including epithelial cells (reviewed in ref. 2; J.B. Rubins and E.N. Janoff, unpublished data). In this unperturbed setting, IgA-bound organisms also adhere poorly to inflammatory and phagocytic cells. In contrast, once an inflammatory response is initiated, IgA - particularly pIgA - may participate more actively to facilitate killing of $S$. pneumoniae by 2 mechanisms. First, IgA can promote complement binding to organisms. However, whether sufficient levels of complement are available at mucosal sites, either produced by activated phagocytes and epithelial cells or exuded from serum when sufficient tissue injury has occurred, is under investigation. Nevertheless, we have shown a second IgArelated mechanism whereby inflammatory products such as TNF- $\alpha$ and C5a can promote phagocyte activation with IgA-mediated uptake and killing of the pathogen. These data are consistent with the increased expression and affinity of IgA receptors on granulocytes, as well as enhanced phagocytosis (66-71), upon cytokine activation. Complement may participate directly through $\mathrm{C} 3$ bi bound to the organism to facilitate binding, uptake, and possibly killing of $\operatorname{IgA}$-opsonized $S$. pneumoniae by phagocytes. Moreover, complement products such as C5a may serve indirectly to activate phagocytes and promote killing of organisms opsonized with immune IgA alone.

In summary, we have shown that capsule-specific IgA, particularly PIgA, mediates uptake and killing of S. pneumoniae in the presence of an intact alternative complement pathway, or in the absence of this pathway, with activated phagocytes. In contrast to IgG, the distinct inability of IgA to mediate appreciable phagocyte interactions in the absence of complement or inflammation highlights the more passive protective role of IgA in immune exclusion. However, IgA, particularly pIgA, may participate actively in protective phagocytosis and killing of $S$. pneumoniae once the inflammatory process is initiated.

\section{Acknowledgments}

The authors thank Barbara Benson and Darlene Charboneau for technical expertise; Ann Emery for secretarial support; Jill G. Hackell and Lederle-Praxis Biologicals, Pearl River, New York for providing PNU-IMMUNE vaccine; and William Latham, Massachusetts Department of Health, for purified tetanus toxoid. This work was supported by National Institutes of Health grants AI-31373, AI-39445, AI-042240, DE-42600, and DE-72621, and the Department of Veterans Affairs Research Service.

1. Svanborg-Eden, C., and Svennerholm, A.-M. 1978. Secretory immunoglobulin A and $\mathrm{G}$ antibodies prevent adhesion of Escherichia coli to human urinary tract epithelial cells. Infect. Immun. 22:790-797.

2. Russell, M.W., Kilian, M., and Lamm, M.E. 1999. Biological activities of IgA. In Mucosal immunology. 2nd edition. P.L. Ogra et al., editors. Academic Press. San Diego, CA. 225-240.

3. Underdown, B.J., and Schiff, J.M. 1986. Immunoglobulin A: strategic defense initiative at the mucosal surface. Annu. Rev. Immunol. 4:389-417.

4. Kilian, M., Mestecky, J., and Russell, M.W. 1988. Defense mechanisms involv- ing Fc-dependent functions of immunoglobulin A and their subversion by bacterial immunoglobulin A proteases. Microbiol. Rev. 52:296-303.

5. Mestecky, J., and McGhee, J.R. 1987. Immunoglobulin A (IgA): molecular and cellular interactions involved in IgA biosynthesis and immune response. In Advances in immunology. Volume 40. F.J. Dixon, editor. Academic Press. San Diego, CA. 153-245.

6. McGhee, J.R., et al. 1992. The mucosal immune system: from fundamental concepts to vaccine development. Vaccine. 10:75-88.

7. Johnson, S., Gerding, D.N., and Janoff, E.N. 1992. Systemic and mucosal antibody responses to toxin A in patients infected with Clostridium difficile (CD) infection. J. Infect. Dis. 166:1287-1294.

8. Johnson, S., Sypura, W.D., Gerding, D.N., Ewing, S., and Janoff, E.N. 1995. Selective neutralization of a bacterial enterotoxin by serum immunoglobulin A in response to mucosal disease. Infect. Immun. 63:3166-3173.

9. Kerr, M.A. 1990. The structure and function of human IgA. Biochem. J. 271:285-296.

10. Mazanec, M.B., Kaetzel, C.S., Lamm, M.E., Fletcher, D., and Nedrud, J.G. 1992. Intracellular neutralization of virus by immunoglobulin A antibodies. Proc. Natl. Acad. Sci. USA. 89:6901-6905.

11. Kaetzel, C.S., Robinson, J.K., Chintalacharuvu, K.R., Vaerman, J.-P., and Lamm, M.E. 1991. The polymeric immunoglobulin receptor (secretory component) mediates transport of immune complexes across epithelial cells: A local defense function for IgA. Proc. Natl. Acad. Sci. USA. 88:8796-8800.

12. Russell, M.W., Reinholdt, J., and Kilian, M. 1989. Anti-inflammatory activity of human IgA antibodies and their Fab $\alpha$ fragments: inhibition of IgGmediated complement activation. Eur. J. Immunol. 19:2243-2249.

13. Jarvis, G.A., and Griffiss, J.M. 1991. Human IgA1 blockade of IgG-initiated lysis of Neisseria meningitidis is a function of Fab binding to the polysaccharide capsule. J. Immunol. 147:1962-1967.

14. Carson, P.J., Schut, R.L., Simpson, M.L., O’Brien, J., and Janoff, E.N. 1995. Antibody class and subclass responses to pneumococcal polysaccharides following immunization of human immunodeficiency virus-infected patients. J. Infect. Dis. 172:340-345.

15. Johnson, S., Opstad, N.L., Douglas, J.M.,Jr., and Janoff, E.N. 1996. Prolonged and preferential production of polymeric immunoglobulin $\mathrm{A}$ in response to Streptococcus pneumoniae capsular polysaccharides. Infect. Immun. 64:4339-4344.

16. Tuomanen, E.I., Austrian, R., and Masure, H.R. 1995. Pathogenesis of pneumococcal infection. N. Engl. J. Med. 332:1280-1284.

17. Musher, D.M., et al. 1992. Antibody to capsular polysaccharides of Streptococcus pneumoniae: prevalence, persistence, and response to revaccination. Clin. Infect. Dis. 17:66-73.

18. Bennett, N.M., Buffington, J., and La Force, F.M. 1992. Pneumococcal bacteremia in Monroe County, New York. Am. J. Public Health. 82:1513-1516.

19. Austrian, R., and Gold, J. 1964. Pneumococcal bacteremia with especial reference to bacteremic pneumococcal pneumonia. Ann. Intern. Med. 60:759-776.

20. Lue, C., Tarkowski, A., and Mestecky, J. 1988. Systemic immunization with pneumococcal polysaccharide vaccine induces a predominant IgA2 response of peripheral blood lymphocytes and increases of both serum and secretory anti-pneumococcal antibodies. J. Immunol. 140:3793-3800.

21. Tarkowski, A., et al. 1990. Immunization of humans with polysaccharide vaccines induces systemic, predominantly polymeric IgA2-subclass antibody responses. J. Immunol. 144:3770-3778.

22. Opstad, N.L., et al. 1995. Impact of Streptococcus pneumoniae bacteremia and human immunodeficiency virus type 1 on oral mucosal immunity. J. Infect. Dis. 172:567-571.

23. Weiss, P.J., Wallace, M.R., Oldfield, E.C., III., O’Brien, J., and Janoff, E.N. 1995. Response of recent human immunodeficiency virus seroconverters to the pneumococcal polysaccharide vaccine and Haemophilus influenzae type b conjugate vaccine. J. Infect. Dis. 171:1217-1222.

24. Friguet, B., Chaffotte, A.F., Djavadi-Ohaniance, L., and Goldberg, M.E. 1985. Measurements of the true affinity constant in solution of antigen-antibody complexes by enzyme-linked immunosorbent assay. J. Immunol. Methods. 77:305-319.

25. Janoff, E.N., Hardy, W.D., Smith, P.D., and Wahl, S.M. 1991. Levels, specificity, and affinity of IgG specific for recall antigens in patients with HIV.J. Immunol. 147:2130-2135.

26. Kutteh, W.H., Koopman, W.J., Conley, M.E., Egan, M.L., and Mestecky, J. 1980. Production of predominantly polymeric IgA by human peripheral blood lymphocytes stimulated in vitro with mitogens. J. Exp. Med. 152:1424-1429.

27. Schrohenloher, R.E., Kunkel, H.G., and Tomasi, T.B. 1964. Activity of dissociated and reassociated 19S anti--globulins. J. Exp. Med. 120:1215-1229.

28. Negro Ponzi, A., Merlino, C., Angeretti, A., and Penna, R. 1985. Virus-specific polymeric immunoglobulin A antibodies in serum from patients with rubella, measles, varicella, and herpes zoster virus infections. J. Clin. Microbiol. 22:505-509.

29. Gray, B.M. 1990. Opsonophagocidal activity in sera from infants and children immunized with Haemophilus influenzae capsular polysaccharides. Infect. Immun. 85:694-697.

30. Romero-Steiner, S., et al. 1997. Standardization of an opsonophagocytic 
assay for the measurement of functional activity against Streptococcus pnenmoniae using differentiated HL-60 cells. Clin. Diagn. Lab. Immunol. 4:415-422.

31. Janoff, E.N., Fasching, C., Ojoo, J.C., O’Brien, J., and Gilks, C.F. 1997. Responsiveness of HIV-1-infected Kenyan women with and without pneumococca disease to pneumococcal vaccine. J. Infect. Dis. 175:975-978.

32. Skubitz, K.M., Zhen, Y., and August, J.T. 1984. Dexamethasone synergistically induces chemotactic peptide receptor expression in HL-60 cells. Blood. 59:586-593

33. Platt-Mills, T.A.E., and Ishizaka, K. 1974. Activation of the alternative pathway of human complement by rabbit cells. J. Immunol. 113:348-358.

34. Polotsky, Y., Nataro, J.P., Kotler, D., Barrett, T.J., and Orenstein, J.M. 1997. HEp-2 cell adherence patterns, serotyping, and DNA analysis of Escherichia coli isolates from eight patients with AIDS and chronic diarrhea. J. Clin. Microbiol. 35:1952-1958.

35. Heilmann, C., Barington, T., and Sigsgaard, T. 1988. Subclass of individual IgA-secreting human lymphocytes. Investigation of in vivo pneumococcal polysaccharide-induced and in vitro mitogen-induced blood B cells by monolayer plaque-forming cell assays. J. Immunol. 140:1496-1499.

36. Payne, N.R., Concepcion, N.F., and Anthony, B.F. 1990. Opsonic effect of jacalin and human immunoglobulin A on type II group B stretotocci. Infect. Immun. 58:3663-3670.

37. Russell, G.J., Gotschlich, E.C., and Blake, M.S. 1984. A surface receptor specific for human IgA on group B streptococci possessing the Ibc protein antigen. J. Exp. Med. 160:1467-1475.

38. Heden, L.O., Frithz, E., and Lindahl, G. 1991. Molecular characterization of an IgA receptor from group B streptococci: sequence of the gene, identification of a proline-rich region with unique structure and isolation of $\mathrm{N}$-terminal fragments with IgA-binding capacity. Eur. J. Immunol. 21:1481-1490

39. Hammerschmidt, S., Talay, S.R., Brandtzaeg, P., and Chhatwal, G.S. 1997. SpsA, a novel pneumococcal surface protein with specific binding to secretory immunoglobulin A and secretory component. Mol. Microbiol. 25:1113-1124.

40. Rosenow, C., et al. 1997. Contribution of novel choline-binding proteins to adherence, colonization and immunogenicity of Streptococcus pneumoniae. Mol. Microbiol. 25:819-829.

41. Bessen, D., and Fischetti, V.A. 1988. Passive acquired mucosal immunity to group A streptococci by secretory immunoglobulin A. J. Exp. Med. 167:1945-1950.

42. Steinitz, M., Tamir, S., Ferne, M., and Goldfarb, A. 1986. A protective human monoclonal IgA antibody produced in vitro: anti-pneumococcal antibody engendered by Epstein-Barr virus-immortalized cell line. Eur. J. Immunol. 16:187-193

43. Hiemstra, P.S., Gorter, A., Stuurman, M.E., van Es, L.A., and Daha, M.R 1987. Activation of the alternative pathway of complement by human serum IgA. Eur. J. Immunol. 17:321-326.

44. Schaapherder, A.F.M., Gooszen, H.G., te Bulte, M.-T.J.W., and Daha, M.R 1995. Human complement activation via the alternative pathway on porcine endothelium initiated by IgA antibodies. Transplantation. 60:287-291.

45. Valim, Y.M.L., and Lachmann, P.J. 1991. The effect of antibody isotype and antigenic epitope density on the complement-fixing activity of immune complexes: a systematic study using chimaeric anti-NIP antibodies with human Fc regions. Clin. Exp. Immunol. 84:1-8.

46. Renegar, K.B., Jackson, G.D.F., and Mestecky, J. 1998. In vitro comparison of the biologic activities of monoclonal monomeric IgA, polymeric IgA, and secretory IgA. J. Immunol. 160:1219-1223.

47. Taylor, H.P., and Dimmock, N.J. 1985. Mechanism of neutralization of influenza virus by secretory IgA is different from that of monomeric IgA or IgG. J. Exp. Med. 161:198-209.

48. Duncan, A.R., and Winter, G. 1998. The binding site for C1q on IgG. Nature. 332:738-740.

49. Sørensen, V., Sundvold, V., Michaelsen, T.E., and Sandlie, I. 1999. Polymerization of IgA and IgM: roles of $\mathrm{Cys}^{309} / \mathrm{Cys}^{414}$ and the secretory tailpiece. J. Immunol. 162:3448-3455.

50. Hostetter, M.K., Krueger, R.A., and Schmeling, D.J. 1984. The biochemistry of opsonization: central role of the reactive thiolester of the third component of complement. J. Infect. Dis. 150:653-661.

51. Hostetter, M.K. 1986. Serotypic variations among virulent pneumococci in deposition and degradation of covalently bound C3b: implications for phagocytosis and antibody production. J. Infect. Dis. 153:682-693.

52. Reynolds, H.Y. 1987. Bronchoalveolar lavage. Am. Rev. Respir. Dis. 135:250-263.

53. Kaplan, M.E., Dalmasso, A.P., and Woodson, M. 1972. Complementdependent opsonization of incompatible erythrocytes by human secretory IgA. J. Immunol. 108:275-278.

54. Hiemstra, P.S., et al. 1988. Activation of complement by serum IgA, secretory IgA, and IgA1 fragments. Mol. Immunol. 25:527-533.

55. Russell, M.W., and Mansa, B. 1989. Complement-fixing properties of human IgA antibodies. Alternative pathway complement activation by plasticbound, but not specific antigen-bound. Scand. J. Immunol. 30:175-183.

56. Zhang, W., and Lachman, P.J. 1994. Glycosylation of IgA is required for optimal activation of the alternative complement pathway by immune complexes. Immunology. 81:137-141.

57. Miletic, V.D., and Frank, M.M. 1995. Complement-immunoglobulin interactions. Curr. Opin. Immunol. 7:41-47.

58. Lappin, D.F., Birnie, G.D., and Whaley, K. 1990. Interferon-mediated transcriptional and post-transcriptional modulation of complement gene expression in human monocytes. Eur. J. Biochem. 194:177-184.

59. Hetland, G., Johnson, E., Falk, R.J., and Eskeland, T. 1986. Synthesis of complement components $\mathrm{C} 5, \mathrm{C} 6, \mathrm{C} 7, \mathrm{C} 8$ and $\mathrm{C} 9$ in vitro by human monocytes and assembly of the terminal complement complex. Scand. J. Immunol. 24:421-428.

60. Einstein, L.P., Schneeberger, E.E., and Colten, H.R. 1976. Synthesis of the second component of complement by long-term primary cultures of human monocytes. J. Exp. Med. 143:114-126.

61. Whaley, K. 1980. Biosynthesis of the complement components and the regulatory proteins of the alternative complement pathway by human peripheral blood monocytes. J. Exp. Med. 151:501-516.

62. Cole, F.S., Matthews, W.J., Jr., Marino, J.T., Gash, D.J., and Colton, H.R. 1980. Control of complement synthesis and secretion in bronchoalveolar and peritoneal macrophages. J. Immunol. 125:1120-1124.

63. Andoh, A., Fujiyama, Y., Bamba, T., and Hosoda, S. 1993. Differential cytokine regulation of complement $\mathrm{C} 3, \mathrm{C} 4$, and factor $\mathrm{B}$ synthesis in human intestinal epithelial cell line, Caco-2.J. Immunol. 151:4239-4247.

64. Rothman, B.L., Despins, A.W., and Kreutzer, D.L. 1990. Cytokine regulation of C3 and C5 production by the human type II pneumocyte cell line, A549. J. Immunol. 145:592-598.

65. Botto, M., Lissandrini, D., Sorio, C., and Walport, M.J. 1992. Biosynthesis and secretion of complement component (C3) by activated human polymorphonuclear leukocytes. J. Immunol. 149:1348-1355.

66. Nikolova, E.B., and Russell, M.W. 1995. Dual function of human IgA antibodies: inhibition of phagocytosis in circulating neutrophils and enhancement of responses in IL-8-stimulated cells. J. Leukoc. Biol. 57:875-882.

67. Hostoffer, R.W., Krukovets, I., and Berger, M. 1994. Enhancement by tumor necrosis factor- $\alpha$ of Fc $\alpha$ receptor expression and IgA-mediated superoxide generation and killing of Pseudomonas aeruginosa by polymorphonuclear leukocytes. J. Infect. Dis. 170:82-87.

68. Bobak, D.A., Gaither, T.A., Frank, M.M., and Tenner, A.J. 1987. Modulation of FcR function by complement: subcomponent C1q enhances the phagocytosis of IgG-opsonized targets by human monocytes and culture-derived macrophages. J. Immunol. 138:1150-1156.

69. Klebanoff, S.J., et al. 1986. Stimulation of neutrophils by tumor necrosis factor. J. Immunol. 136:4220-4229.

70. Weisbart, R.H., Kacena, A., Schuh, A., and Golde, D.W. 1988. GM-CSF induces human neutrophil IgA-mediated phagocytosis by an IgA Fc receptor activation mechanism. Nature. 332:647-648.

71. Hostoffer, R.W., Krukovets, I., and Berger, M. 1993. Increased Fc $\alpha$ R expression and IgA-mediated function on neutrophils induced by chemoattractants. J. Immunol. 150:4532-4540. 